\title{
Solid-Gas Coupling Model for Coal-Rock Mass Deformation and Pressure Relief Gas Flow in Protection Layer Mining
}

\author{
Zhuohui Zhu $\mathbb{D}^{1,2,3}$ Tao Feng, ${ }^{1,2,3}$ Zhigang Yuan, ${ }^{1,2,3}$ Donghai Xie, ${ }^{1,2,3}$ and Wei Chen ${ }^{1}$ \\ ${ }^{1}$ School of Resources, Environment and Safety Engineering, Hunan University of Science and Technology, Xiangtan, \\ Hunan 411201, China \\ ${ }^{2}$ Hunan Provincial Key Laboratory of Safe Mining Techniques of Coal Mines, Hunan University of Science and Technology, \\ Xiangtan, Hunan 411201, China \\ ${ }^{3}$ Work Safety Key Lab on Prevention and Control of Gas and Roof Disasters for Southern Coal Mines, \\ Hunan University of Science and Technology, Xiangtan, Hunan 411201, China
}

Correspondence should be addressed to Zhuohui Zhu; zhuzhuohui1982@qq.com

Received 28 November 2017; Accepted 10 January 2018; Published 2 April 2018

Academic Editor: Hang Lin

Copyright (C) 2018 Zhu Zhuohui et al. This is an open access article distributed under the Creative Commons Attribution License, which permits unrestricted use, distribution, and reproduction in any medium, provided the original work is properly cited.

The solid-gas coupling model for mining coal-rock mass deformation and pressure relief gas flow in protection layer mining is the key to determine deformation of coal-rock mass and migration law of pressure relief gas of protection layer mining in outburst coal seams. Based on the physical coupling process between coal-rock mass deformation and pressure-relief gas migration, the coupling variable of mining coal-rock mass, a part of governing equations of gas seepage field and deformation field in mining coal-rock mass, is introduced. Then, a new solid-gas coupling mathematical model reflecting the coupling effects of gas adsorption/desorption, gas pressure, and coal-rock mass deformation on the mining coal-rock mass deformation and pressure relief gas flow is established combined with the corresponding definite conditions. It lays a theoretical foundation for the numerical calculation of the deformation of mining coal-rock mass and the migration law of gas under pressure relief in the outburst coal seam group.

\section{Basic Hypothesis}

After mining protection seam, the initial rock stress balance is broken and the coal-rock mass deforms, which destroys the dynamic balance of original gas adsorption and desorption, and part of gas desorbs and diffuses to the mining fissure and gas pressure reduces, which makes the coal-rock mass pressure state changed; namely, deformation of mining coal-rock mass and flow of pressure relief gas are a multiphysical field coupling process. Therefore, the establishment of a solid-gas coupling mathematical model which can describe the deformation of mining coal-rock mass and pressure relief gas flow is the key to study the deformation of coal-rock mass and the migration law of gas under pressure relief in protection layer mining.

Due to the solid-gas coupling effect of coal-rock mass deformation and pressure relief gas flow are complicated, which involves seepage mechanics, rock mechanics, elasticplastic mechanics and many other subjects [1-5]. The existing research results found that seepage pressure affects the stress field; moreover, the stress field in surrounding rock results in the change of permeability of rock masses $[3,5]$. Based on the previous studies [1-5], the following hypotheses are introduced:

(1) The solid-gas coupling system consists of both solid phase (coal-rock mass) and gas phase.

(2) The solid skeleton deformation of coal-rock mass is small, which can be regarded as homogeneous and isotropic linear elastic medium; the coal seam gas can be regarded as an ideal gas, and its flow process can be regarded as approximately isothermal process, which obeys the Darcy seepage law.

(3) The coal seam is saturated by single-phase gas, and the original gas pressure is uniformly distributed; gas content in coal seam follows the modified Langmuir equation. 
(4) The effective stress of coal-rock mass skeleton follows the modified Terzaghi effective stress principle.

\section{Control Equations of Deformation Field of Mining Coal-Rock Mass}

The control equations of displacement and stress fields of mining coal-rock mass deformation are mainly composed of constitutive equation, geometric equation, and equilibrium equation.

2.1. Constitutive Equation. For the single-phase saturated linear elastic porous media, the effective stress $\sigma_{i i}^{\prime}$ (tension is positive), the pore pressure $p$ (compression is positive), and the total stress $\sigma_{i j}$ follow the modified Terzaghi effective stress principle $[6,7]$ :

$$
E=\sum_{i=1}^{m} S_{i},
$$

where $\delta_{i j}$ is the Kronecker symbol (when $i=j, \delta_{i j}=1$, and when $\left.i \neq j, \delta_{i j}=0\right)$ and $\alpha(\alpha \leq 1)$ is the Biot coefficient, whose value depends on the compressibility of coal-rock mass and is calculated as follows:

$$
\alpha=1-\frac{K}{K_{s}},
$$

where $K$ is the bulk modulus of coal-rock mass skeleton $(\mathrm{Pa})$ and $K_{s}$ is the matrix bulk modulus of coal-rock mass $(\mathrm{Pa})$.

Previous studies have shown that $[8,9]$ adsorption/desorption will cause the bulk strain of coal matrix, and it can be calculated combined with the generalized Hooke's law:

$$
\sigma_{i j}^{\prime}=2 G \varepsilon_{i j}+\frac{2 G v}{1-2 v} \varepsilon_{k k} \delta_{i j}-K \varepsilon_{s} \delta_{i j}
$$

where $v$ is the Poisson's ratio of drainage, $G$ is the shear modulus $(\mathrm{Pa})$, and $\varepsilon_{s}$ is the matrix bulk strain caused by coal adsorption/desorption; the experimental results show that the relationship between $\varepsilon_{s}$ and $p$ meets the Langmuir type curve $[8,9]$ :

$$
\varepsilon_{s}=\varepsilon_{L} \frac{p}{p+p_{L}}
$$

where $\varepsilon_{L}$ is the bulk strain constant when the pore pressure is infinite and $p_{L}$ is the pore pressure when the bulk strain is $0.5 \varepsilon_{L}(\mathrm{~Pa})$.

2.2. Geometric Equation. Assuming that the deformation of coal-rock mass is small, the geometric equation is [10]

$$
\varepsilon_{i j}=\frac{1}{2}\left(u_{i, j}+u_{j, i}\right)
$$

where $u_{i}$ is the displacement in the $i$ direction.

2.3. Equilibrium Equation. According to the virtual displacement principle of elastic mechanics, the momentum equation of coal-rock mass skeleton under quasi-static condition is [11]

$$
\int_{\Omega} \delta \varepsilon_{i j} \sigma_{i j} d V-\int_{\Omega} f_{i} \delta u_{i} d V-\int_{S} \bar{f}_{i} \delta u_{i} d S=0
$$

where $\bar{f}_{i}$ and $f_{i}$ are the boundary surface force and the bulk force of the skeleton, $\delta u_{i}$ and $\delta \varepsilon_{i j}$ are the coordination of virtual displacement variation and virtual strain variation, and $\Omega$ and $S$ are the regions occupied by solid skeleton and the known boundary of stress.

It can be obtained according to (5) and the symmetry of strain tensor [12]:

$$
\delta \varepsilon_{i j}=\delta\left[\frac{1}{2}\left(u_{i, j}+u_{j, i}\right)\right]=\delta u_{i, j} .
$$

Substituting (1) into (6), we get

$\int_{\Omega} \delta \varepsilon_{i j}\left(\sigma_{i j}^{\prime}-\alpha p \delta_{i j}\right) d V-\int_{\Omega} f_{i} \delta u_{i} d V-\int_{S} \bar{f}_{i} \delta u_{i} d S=0$.

According to the Green-Gauss formula, the first item on the left of (8) is

$$
\begin{aligned}
\int_{\Omega} \delta u_{i, j}\left(\sigma_{i j}^{\prime}-\alpha p \delta_{i j}\right) d V= & \int_{S} \delta u_{i}\left(\sigma_{i j}^{\prime}-\alpha p \delta_{i j}\right) n_{j} d S \\
& -\int_{\Omega}\left(\sigma_{i j, j}^{\prime}-\alpha p_{j} \delta_{i j}\right) \delta u_{i} d V .
\end{aligned}
$$

The equilibrium equation of porous medium of coalrock mass can be obtained by (8) and (9):

$$
\sigma_{i j}^{\prime}-\alpha p_{j}+f_{i}=0
$$

Connecting the constitutive equation (3), the geometric equation (5), and the equilibrium equation (10), the governing equations which take the solid-gas coupling deformation field under the effect of coal adsorption/desorption deformation, stress, and pore pressure into account can be obtained:

$$
G u_{i, j j}+\frac{G}{1-2 v} u_{j, j i}-\alpha p_{i}-K \varepsilon_{s, i}+f_{i}=0 .
$$

\section{Governing Equation of Gas Flow in Mining Coal-Rock Mass}

The governing equation of gas flow in coal-rock mass combines the continuity equation, state equation, gas content equation, and Darcy law of motion and eliminates some variables to deduct differential equations. Migration morphology of gas is closely related to the pore structure, but the pore structure of coal seam is complicated; when studying migration law of gas, people are often based on different types of gas diffusion or the primary and secondary roles of seepage to simplify the pore structure of coal seam into pure diffusion pore medium model, pure permeability fracture medium model, and pore-fissure dual-medium model with coexistence of diffusion and permeation. The pore-fissure dual-medium model has been accepted and used by many scholars, and it assumes gas migration in coal 
seam is a continuous process in two steps: diffusion and permeation are a cascade process that the gas diffuses from the matrix to the fissure in the form of diffusion and then flows through the fissures into the tunnel or borehole in the form of the Darcy seepage.

The continuity equation of gas diffusion motion is as follows $[13,14]$ :

$$
\frac{\partial C}{\partial t}=-\nabla m_{c}-Q_{s}
$$

where $m_{c}$ is the mass diffusion flux $\left(\mathrm{kg} /\left(\mathrm{m}^{2} \cdot \mathrm{t}\right)\right), Q_{s}$ is the exchange mass source of the pore system flowing to the fracture system $\left(\mathrm{kg} /\left(\mathrm{m}^{3} \cdot \mathrm{s}\right)\right)$, and $C$ is the quality of adsorbed gas in unit bulk coal:

$$
C=\rho_{g a} \rho_{c} \frac{V_{L} p}{p+p_{L}},
$$

where $\rho_{g a}$ is the gas density under the standard state $\left(\mathrm{kg} / \mathrm{m}^{3}\right)$, $\rho_{c}$ is density of coal-rock mass $\left(\mathrm{kg} / \mathrm{m}^{3}\right)$, and $V_{L}$ is the Langmuir bulk constant $\left(\mathrm{kg} / \mathrm{m}^{3}\right)$.

According to the law of mass conservation, the continuity equation of gas seepage in fracture can be obtained [13-15]:

$$
\frac{\partial\left(\rho_{g} \phi\right)}{\partial t}=-\nabla \cdot\left(\rho_{g} q_{g}\right)+Q_{s}
$$

where $\rho_{g}$ is the gas density $\left(\mathrm{kg} / \mathrm{m}^{3}\right), \phi$ is the porosity of coalrock mass, and $q_{g}$ is the velocity of gas seepage $(\mathrm{m} / \mathrm{s})$.

The gas flow is divided into two open systems: diffusion and seepage. In fact, there is a coupling effect between gas diffusion and seepage, and the adsorption and desorption of gas only change in the state of matter, and it does not cause the change of gas quality in representative bulk units. Therefore, in order to facilitate the analysis and solution, the mass conservation equation of gas flow can be obtained by adding (12) and (14):

$$
\frac{\partial\left(\rho_{g} \phi\right)}{\partial t}+\frac{\partial C}{\partial t}+\nabla \cdot\left(\rho_{g} q_{g}+m_{c}\right)=0
$$

For low permeability coal-rock mass, it can be assumed that the gas is desorbed and diffused into the fracture from the matrix instantaneously, and (15) can be further simplified:

$$
\frac{\partial m}{\partial t}+\nabla \cdot\left(\rho_{g} q_{g}\right)=0
$$

In (16), $m=\rho_{g} \phi+\rho_{g a} \rho_{c}\left(V_{L} p / p+p_{L}\right)$, the first item on the right shows the free gas content in the coal-rock mass, and the second item represents the adsorbed gas content in the coal.

The coal gas, whose flow process could be assumed to be isothermal, is considered as the actual gas, and then the state equation of the gas can be obtained:

$$
\rho_{g}=\frac{M_{g}}{R T} p,
$$

where $M_{g}$ is the molar mass of gas, $R$ is the universal gas constant, and $T$ is the absolute temperature.

The outward coal-rock mass gas flow from the coal seam crack accord with the Darcy seepage law:

$$
q_{g}=-\frac{k}{\mu} \cdot \nabla p
$$

where $k$ is the permeability of the coal seam, $\mu$ is the dynamic viscosity of gas (Pa.s), and $\nabla p$ is the gas pressure gradient $(\mathrm{Pa} / \mathrm{m})$.

Substituting (17) and (18) into (16), we get

$$
\left[\phi+\frac{\rho_{c} p_{a} V_{L} p_{L}}{\left(p+p_{L}\right)^{2}}\right] \frac{\partial p}{\partial t}+p \frac{\partial \phi}{\partial t}-\nabla \cdot\left(\frac{k}{\mu} p \nabla p\right)=0,
$$

where $p_{a}$ is the standard atmospheric pressure (Pa). In (19), the permeability $k$ depends on the porosity of coal $\phi$, and the porosity of coal $\phi$ is a function of the volumetric strain $\varepsilon_{v}$ of coal-rock mass and the volumetric strain $\varepsilon_{s}$ of matrix. Therefore, (11) and (19) are connected by the porosity of coal $\phi$ and physical parameters of permeability $k$.

\section{The Structure of Solid-Gas Coupling Mathematical Model for Coal-Rock Mass Deformation and Pressure Relief Gas Flow in Protection Seam Mining}

4.1. Solid-Gas Coupling Variables in Mining Coal-Rock Mass. Traditional classical percolation mechanics holds that in the pore pressure change process, the solid skeleton of porous media does not produce any elastic-plastic deformation, so the porosity of porous media $\phi$ is a constant. However, under the effect of surrounding rock stress and gas pressure, the solid skeleton of coal will deform in different degrees, which will cause the change of effective stress and lead to the dynamic change of porosity and permeability of coal-rock mass. When it comes to the deformation of coalrock mass in protection layer mining and the solid-gas coupling of pressure relief gas flow, the dynamic changes of porosity, permeability, and other parameters of coal-rock mass must be taken into account. So, it is necessary to establish the theoretical calculation model for the dynamic changes of the basic physical parameters such as porosity and permeability of coal-rock mass.

4.1.1. Dynamic Variation Model of Coal-Rock Mass Porosity. The bulk $V$ of porous medium in coal-rock mass is composed of the bulk of solid phase $V_{s}$ and the bulk of pore volume $V_{p}$, namely, $V=V_{s}+V_{p}$. The definition of porosity is $\phi=V_{p} / V$, and the bulk strain $\varepsilon_{v}$ of coal-rock mass can be obtained by (11) $[16,17]$ :

$$
\begin{gathered}
\varepsilon_{v}=\frac{\Delta V}{V}=-\frac{1}{K}(\bar{\sigma}-\alpha p)+\varepsilon_{s}, \\
\frac{\Delta V_{p}}{V_{p}}=-\frac{1}{K_{p}}(\bar{\sigma}-\beta p)+\varepsilon_{s},
\end{gathered}
$$

where $\bar{\sigma}=-\sigma_{k k} / 3, \beta=1-K_{p} / K_{s}$, and $K_{p}$ is the module of pore bulk.

It is assumed that the strain caused by coal adsorption is equal to the amount of pore variation. Without considering the adsorption effect of gas, the bulk change of porous media meets the equivalent law of Betti-Maxwell: 


$$
\left(\frac{\partial V}{\partial p}\right)_{\bar{\sigma}}=\left(\frac{\partial V_{p}}{\partial \bar{\sigma}}\right)_{p}
$$

and therefore,

$$
K_{p}=\frac{\phi}{\alpha} K .
$$

Then, deducted from the definition of porosity

$$
\begin{gathered}
\frac{\Delta V}{V}=\frac{\Delta V_{s}}{V_{s}}+\frac{\Delta \phi}{1-\phi}, \\
\frac{\Delta V_{p}}{V_{p}}=\frac{\Delta V_{s}}{V_{s}}+\frac{\Delta \phi}{\phi(1-\phi)} .
\end{gathered}
$$

Connecting (20), (21), (24) and (25), we get

$$
\Delta \phi=\phi\left(\frac{1}{K}-\frac{1}{K_{p}}\right)(\bar{\sigma}-p) .
$$

Substituting (20) and (23) into (26), we get

$$
\Delta \phi=(\alpha-\phi)\left(\varepsilon_{v}+\frac{p}{K_{s}}-\varepsilon_{s}\right) .
$$

When the pore pressure of coal-rock mass is $p_{0}$, the initial porosity is $\phi_{0}$, and the initial bulk strain is 0 ; then, the porosity can be expressed as

$$
\phi=\frac{1}{1+S}\left[\left(1+S_{0}\right) \phi_{0}+\alpha\left(S-S_{0}\right)\right] .
$$

In formula (28), $S=\varepsilon_{v}+p / K_{s}-\varepsilon_{s}$ and $S_{0}=p_{0} / K_{s}-\varepsilon_{L} p_{0} /$ $\left(p_{0}+p_{L}\right)$.

4.1.2. Dynamic Change Model of Coal-Rock Mass Permeability. Permeability, a physical parameter reflecting the difficult degree of gas flow in coal seam, is important for studying coupling process between deformation of mining coal-rock mass and gas flow. The relationship between the permeability of coal seam and the bulk strain of coal can be deduced by the Kozeny-Carman equation:

$$
\frac{k}{k_{0}}=\frac{1}{1+\varepsilon_{v}}\left(\frac{\phi}{\phi_{0}}\right)^{3} \text {. }
$$

Neglecting the bulk deformation of coal, (29) can be simplified as

$$
\frac{k}{k_{0}}=\left(\frac{\phi}{\phi_{0}}\right)^{3} .
$$

Connecting (28) and (30), we get

$$
\frac{k}{k_{0}}=\left(\frac{1}{1+S}\left[\left(1+S_{0}\right)+\frac{\alpha}{\phi_{0}}\left(S-S_{0}\right)\right]\right)^{3} .
$$

4.2. Solid-Gas Coupling Mathematical Model of Mining Coal Deformation and Pressure Relief Gas Flow. According to the antecedent, (11) is the control equation of mining coal deformation; substituting (4) into (11), we get

$$
G u_{i, j j}+\frac{G}{1-2 v} u_{j, j i}-\alpha p_{i}-\frac{K \varepsilon_{L} p_{L}}{\left(p+p_{L}\right)^{2}} p_{i}+f_{i}=0 .
$$

It can be obtained from (28):

$$
\frac{\partial \phi}{\partial t}=\frac{\alpha-\phi}{1+S}\left[\frac{\partial \varepsilon_{v}}{\partial t}+\frac{1}{K_{s}} \frac{\partial p}{\partial t}-\frac{\varepsilon_{L} p_{L}}{\left(p+p_{L}\right)^{2}} \frac{\partial p}{\partial t}\right] .
$$

Substituting (33) into (19), the control equation of the pressure relief gas of the mining coal can be obtained:

$$
\begin{gathered}
{\left[\phi+\frac{\rho_{c} p_{a} V_{L} p_{L}}{\left(p+p_{L}\right)^{2}}+\frac{(\alpha-\phi) p}{(1+S) K_{s}}-\frac{(\alpha-\phi) \varepsilon_{L} p_{L} p}{(1+S)\left(p+p_{L}\right)^{2}}\right] \frac{\partial p}{\partial t}} \\
-\nabla \cdot\left(\frac{k}{\mu} p \nabla p\right)=-\frac{(\alpha-\phi) p}{(1+S)} \frac{\partial \varepsilon_{v}}{\partial t} .
\end{gathered}
$$

Formulas (32) and (34) constitute a gas-solid coupling mathematical model of coal seam deformation and pressure relief gas flow in protection seam mining.

4.3. Solid-Gas Coupling Mathematical Model of Rock Mass Deformation and Pressure Relief Gas Flow in Protective Seam Mining. When the rock mass is affected by mining, deformation and cracks occur, the change of rock porosity will lead to the change of permeability, and the pressure relief gas will flow under the effect of gas pressure gradient. The flow of gas in the mining rock mass is different from that of the mining coal, mainly in the absence of adsorption of the rock mass on the gas. Therefore, there is no need to consider the deformation of bulk expansion caused by the gas diffusion process and gas adsorption/desorption, so it is necessary to modify the solid-gas coupling mathematical model of mining coal deformation and pressure relief gas flow.

To sum up, the governing equation of mining rock mass deformation can be expressed as

$$
G u_{i, j j}+\frac{G}{1-2 v} u_{j, j i}-\alpha p_{i}+f_{i}=0 .
$$

Similarly, the control equation of pressure relief gas of mining coal can be expressed as

$$
\left[\phi+\frac{(\alpha-\phi) p}{\left(1+S_{1}\right) K_{s}}\right] \frac{\partial p}{\partial t}-\nabla \cdot\left(\frac{k}{\mu} p \nabla p\right)=-\frac{(\alpha-\phi) p}{\left(1+S_{1}\right)} \frac{\partial \varepsilon_{v}}{\partial t},
$$

where $S_{1}=\varepsilon_{v}+p / K_{s}$.

4.4. Definite Conditions. The control equations (32) and (34-36) constitute the complicated solid-gas coupling mathematical model of the mining coal-rock mass deformation and the pressure relief gas flow. For a particular solution, additional conditions must be added, that is, initial conditions and boundary conditions. Definite conditions of the model include the boundary conditions of the deformation field of the mining coal-rock mass and the initial conditions and boundary conditions of the gas seepage [17-22]. 
4.4.1. Boundary Conditions of Mining Coal-Rock Mass Deformation Field. The first kind of boundary condition: the surface force of the solid skeleton of the coal-rock mass is known as

$$
\sigma_{i j} \cdot n_{j}=\bar{f}_{i}(x, y, z, t), \quad t \in[0,+\infty) .
$$

The second kind of boundary condition: the surface displacement of the solid skeleton of the coal-rock mass is known as

$$
u_{i}(x, y, z, t)=\overline{g_{i}}(x, y, z, t), \quad t \in[0,+\infty),
$$

where $n_{j}$ is the unit normal vector of the surface solid skeleton of coal-rock mass and $\bar{g}_{i}$ and $\bar{f}_{i}$ are the known surface displacement functions and known surface stress distribution functions.

\subsubsection{Initial Conditions and Boundary Conditions of Gas Seepage}

(1) Initial Conditions of Gas Seepage

$$
\begin{aligned}
\left.p(x, y, z)\right|_{t=0} & =\bar{p}(x, y, z), \\
\left.q(x, y, z)\right|_{t=0} & =\bar{q}(x, y, z) .
\end{aligned}
$$

(2) Boundary Conditions of Gas Seepage. The first kind of boundary condition (the Dirichlet condition):

$$
p(x, y, z, t)=\bar{p}(x, y, z, t), \quad t \in[0,+\infty) .
$$

The second kind of boundary condition (the Neumann condition):

$$
\frac{\partial p(x, y, z, t)}{\partial n}=\bar{Q}(x, y, z, t), \quad t \in[0,+\infty) .
$$

The third kind of boundary condition (equal flow at the interface):

$$
\left.K \frac{\partial p}{\partial n}\right|_{1}=\left.K^{\prime} \frac{\partial p^{\prime}}{\partial n^{\prime}}\right|_{2}
$$

where $\bar{p}$ and $\bar{Q}$ are the distribution function and the fluid flow function of the pressure $p$ and time $t$ at the point $(x, y, z)$ of the outer boundary, respectively, and $\bar{q}$ is the initial flow value at the outer boundary.

\section{Conclusions}

The solid-gas coupling effect of coal-rock mass deformation and pressure relief gas flow in protective layer mining is very complicated, involving rock mechanics, seepage mechanics, elastic mechanics, and other subjects. Based on the existing research results, the seepage control equation of mining coalrock mass and the control equation of deformation field are established including solid-gas coupling variable of mining coal-rock mass, namely, the dynamic change equation of coalrock mass porosity and permeability. According to the coupling equations, the corresponding definite conditions are put forward, and the solid-gas coupling mathematical model of the deformation of coal-rock mass and pressure relief gas flow in the protection layer mining are built. The details are as follows:
(1) The control equations (32) and (35) of the coal-rock mass deformation in protection layer mining that contain the coupling term which reflects the pore gas pressure and gas adsorption/desorption effect is established, which can only be solved by connecting with the governing equations of gas seepage field.

(2) The governing equations (34) and (36) of the flow field of the pressure relief gas flow in the mining coal-rock mass that include the coupling term reflecting the effect of coal-rock mass deformation is established, and it is necessary to connect with the control equation of mining coal-rock deformation field to be solved.

(3) The combination of the solid-gas coupling control equation of mining coal-rock mass deformation and pressure relief gas flow with definite conditions is established, which constitute the solid-gas coupling mathematical model of coal-rock mass deformation and pressure relief gas flow, and it lays a foundation for numerical simulation research of coal-rock mass deformation and pressure relief gas flow law in following protection layer mining. Numerical simulations research will be showed in other papers.

\section{Conflicts of Interest}

The authors declare that there are no conflicts of interest regarding the publication of this paper.

\section{Acknowledgments}

This study was partially supported by the National Natural Science Foundation of China (51774131 and 51604111), the Natural Science Foundation of Hunan Province (2017JJ2082), and the Doctoral Scientific Research Foundation (E51778).

\section{References}

[1] S. Zhou and J. Sun, "Coal seam gas flow theory and its application," International Journal of Coal Science \& Technology, vol. 2, no. 1, pp. 24-37, 1965.

[2] P. Sun, "Study of flow in gas flow fields of coal seams," International Journal of Coal Science \& Technology, vol. 12, no. 4, pp. 74-82, 1987.

[3] Y. Zhao, L. Zhang, W. Wang, J. Tang, H. Lin, and W. Wan, "Transient pulse test and morphological analysis of single rock fractures," International Journal of Rock Mechanics \& Mining Sciences, vol. 91, pp. 139-154, 2017.

[4] W. H. Somerton, I. M. Soylemezoglu, and R. C. Dudley, "Effect of stress on permeability of coal," International Journal of Rock Mechanics and Mining Sciences \& Geomechanics Abstracts, vol. 12, no. 5-6, pp. 129-145, 1975.

[5] Y. Zhao, J. Tang, Y. Chen, L. Zhang, W. Wang, and J. Liao, "Hydromechanical coupling tests for mechanical and permeability characteristics of fractured limestone in complete stress-strain process," Environmental Earth Sciences, vol. 76, no. 1, pp. 1-18, 2016.

[6] G. Hu, H. Wang, and Z. Yuan, "Discrimination criterion of ultimate gas pressure of protection region for exploiting protective layer," International Journal of Coal Science and Technology, vol. 7, pp. 1131-1136, 2010. 
[7] P. Sun, Introduction to Coupling Model for Multiphysics and Numerical Simulations, China Science and Technology Press, Beijing, China, 2007.

[8] J. Zhou, W. Wang, Y. Jiang et al., "A permeability model including effective stress and coal matrix shrinking effect," Rock and Soil Mechanics, vol. 7, pp. 2317-2323, 2010.

[9] G. Yin, Gas-Solid Coupling Instability Theory of Gas-Bearing Coal, Rock and Study, Science Press, Beijing, China, 2011.

[10] W. Zhu, "Coupled thermal-hydraulic-mechanical model during rock damage and its preliminary application," Rock and Soil Mechanics, vol. 12, pp. 3851-3857, 2009.

[11] X. Wang, Basic Principle of Finite Element Method and Numerical Methods, Tsinghua University Press, Beijing, China, 2003.

[12] H. Lin, W. Xiong, Z. Xiong, and F. Gong, "Three-dimensional effects in a flattened Brazilian disk test," International Journal of Rock Mechanics and Mining Sciences, vol. 74, pp. 10-14, 2015.

[13] Y. S. Zhao, H. Z. Qing, and Q. Z. Bai, "Mathematical model for solid-gas coupled problems on the methane flowing in coal seam," Acta Mechanica Solida Sinica, vol. 6, no. 4, p. 459, 1993.

[14] S. Valliappan and W. H. Zhang, "Numerical modeling of methane gas migration in dry coal seams," Geomechanics Abstracts, vol. 1, p. 10, 1997.

[15] G. Hu, "Research on a dynamically coupled deformation and gas flow model applied to low-permeability coal," Journal of China University of Mining and Technology, vol. 1, pp. 1-6, 2011.

[16] H. Liu, "Fully coupled model and engineering application for deformation and pressure-relief gas flow of remote coal and rock mass due to mining," Doctoral dissertation, China University of Mining and Technology, Xuzhou, China, 2010.

[17] Y. Cheng, H. Liu, P. Guo et al., "A theoretical model and evolution characteristic of mining-enhanced permeability in deeper gassy coal seam," Journal of China Coal Society, vol. 39, no. 8, pp. 1650-1658, 2014.

[18] W. Zhu, C. Wei, F. Zhang et al., "Investigation of water inrush from karst subsidence column by using a coupled hydromechanical model," Chinese Journal of Underground Space and Engineering, vol. 7, no. 5, pp. 928-933, 2009.

[19] Z. Zhu, Research on Outburst Prevention Mechanism and Protective Effect of Protection Layer Mining: Near Distance of Gently Inclined Coal Seam Group, Ph.D. thesis, Central South University, Changsha, China, 2016.

[20] S. Harpalain, "Gas flow through stressed coal," Ph.D. thesis, Berkeley University of California, Berkeley, CA, USA, 1985.

[21] Y. Zhao, S. Luo, Y. Wang, W. Wang, L. Zhang, and W. Wan, "Numerical analysis of karst water inrush and a criterion for establishing the width of water-resistant rock pillars," Mine Water and the Environment, vol. 76, pp. 1-18, 2017.

[22] Y. Zhao, Y. Hu, B. Zhao, and D. Yang, "Nonlinear coupled mathematical model for solid deformation and gas seepage in fractured media," Transport in Porous Media, vol. 55, no. 2, pp. 119-136, 2004. 


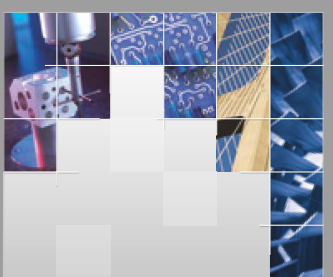

\section{Enfincering}
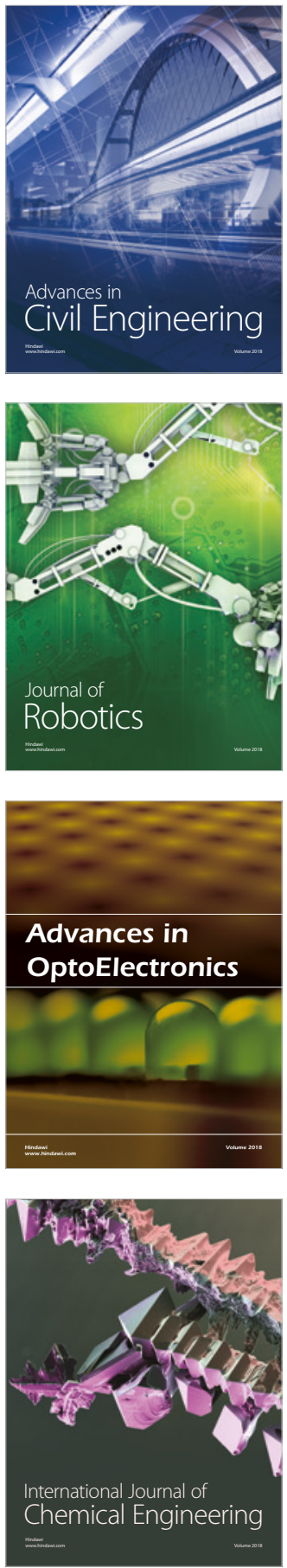

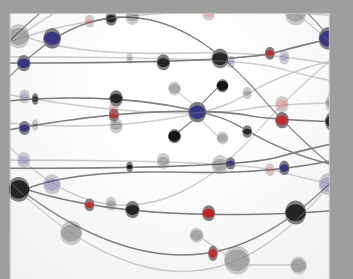

\section{Rotating \\ Machinery}

The Scientific World Journal

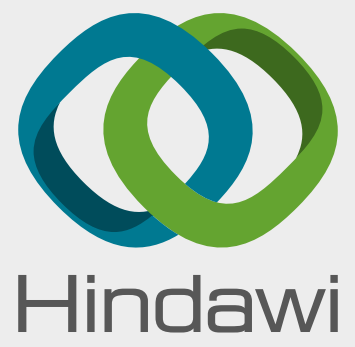

Submit your manuscripts at

www.hindawi.com
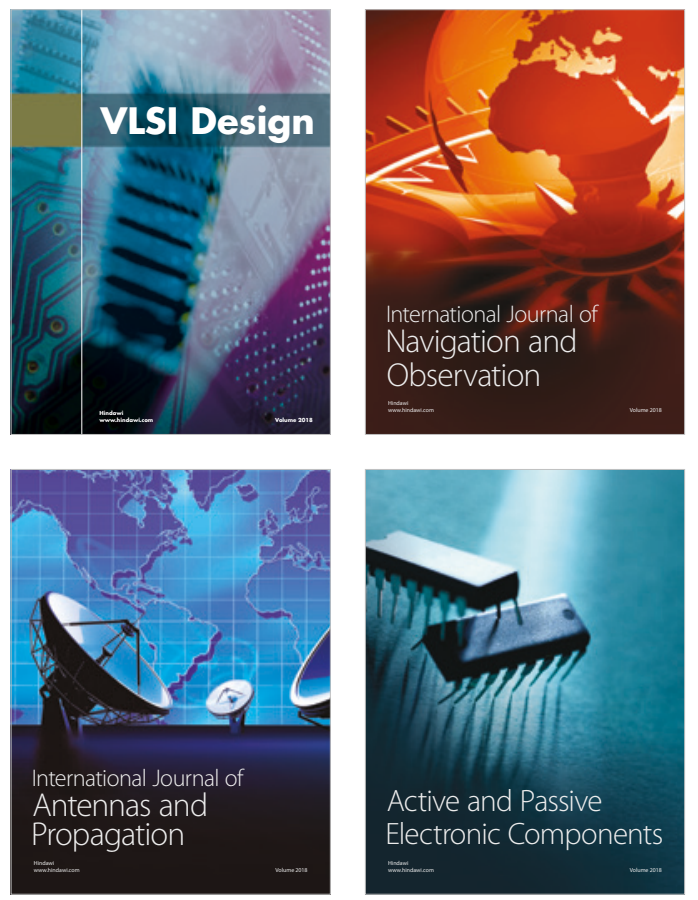
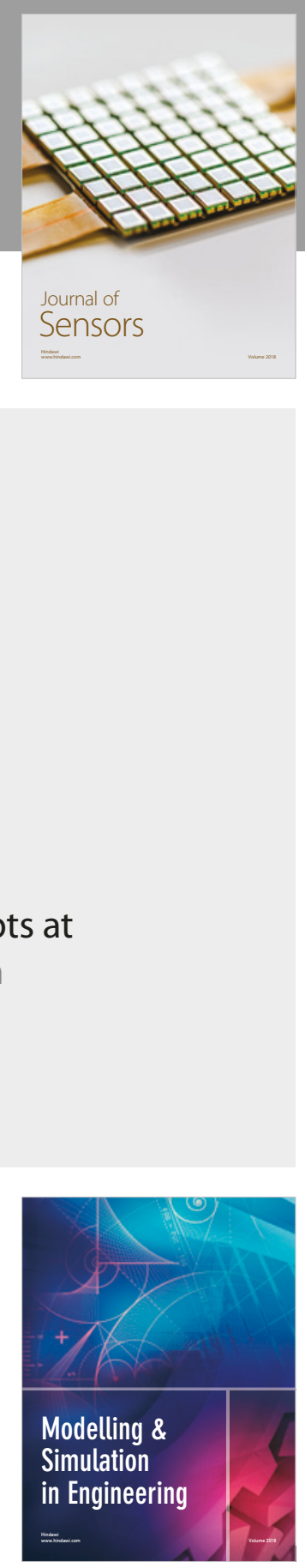

\section{Advances \\ Multimedia}
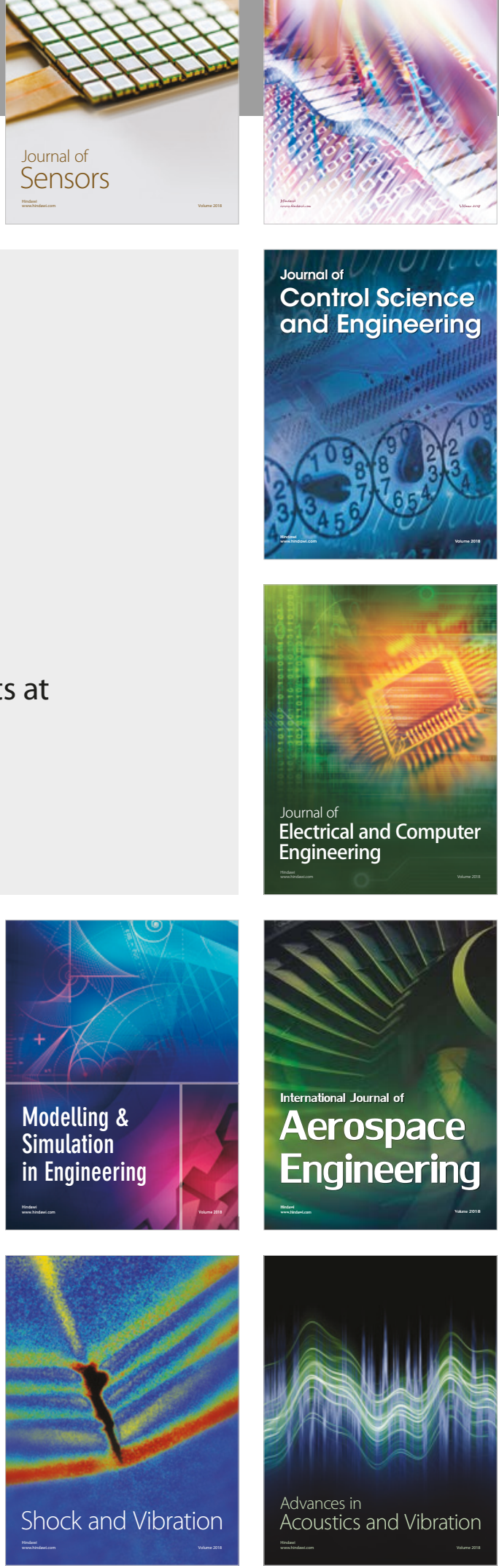